\title{
Towards a Rational Design of Zeolite-Polymer Composite Nanofibers for Efficient Adsorption of Creatinine
}

\author{
Ryo Takai, ${ }^{1,2}$ Rio Kurimoto,, ${ }^{1,2}$ Yasuhiro Nakagawa, ${ }^{1,2}$ Yohei Kotsuchibashi, \\ Koki Namekawa, ${ }^{1}$ and Mitsuhiro Ebara ${ }^{1,2,3}$ \\ ${ }^{1}$ International Center for Materials Nanoarchitectonics (WPI-MANA), National Institute for Materials Science (NIMS), Ibaraki, Japan \\ ${ }^{2}$ Graduate School of Pure and Applied Science, University of Tsukuba, Ibaraki, Japan \\ ${ }^{3}$ Graduate School of Industrial Science and Technology, Tokyo University of Science, Tokyo, Japan
}

Correspondence should be addressed to Mitsuhiro Ebara; ebara.mitsuhiro@nims.go.jp

Received 15 June 2016; Revised 23 August 2016; Accepted 4 September 2016

Academic Editor: Zeeshan Khatri

Copyright (C) 2016 Ryo Takai et al. This is an open access article distributed under the Creative Commons Attribution License, which permits unrestricted use, distribution, and reproduction in any medium, provided the original work is properly cited.

\begin{abstract}
This report describes the compositional and structural design strategy of a zeolite-polymer composite nanofiber mesh for the efficient removal of uremic toxins towards blood purification application. The nanofiber is fabricated by electrospinning composite solution of biocompatible poly(ethylene-co-vinyl alcohol) (EVOH) and zeolite particles which are capable of selectively adsorbing uremic toxins such as creatinine. By controlling electrospinning conditions carefully, the incorporated zeolites in EVOH were found to correspond closely to the feed ratios. Elemental mapping images of Si show that zeolites were uniformly blended within the fibers. The fabricated composite fibers successfully adsorbed creatinine from solution and the adsorption capacity reached a maximum at $12 \mathrm{~h}$. The crystallinity of the nanofiber was also controlled by varying the composition of ethylene content in EVOH. Less crystallinity resulted in higher creatinine adsorption capacity due to the barrier property of EVOH. Cytotoxicity assay demonstrated that the composite fibers showed less toxicity than free zeolite particles which killed more than $95 \%$ of cells. The proposed composite fibers, therefore, have the potential to be utilized as a new approach to removing creatinine selectively from the bloodstream.
\end{abstract}

\section{Introduction}

Chronic kidney disease (CKD) is a worldwide public health problem. About $10 \%$ of the population worldwide is affected by CKD $[1,2]$, and millions die each year because they do not have access to affordable treatment [3]. The main function of kidneys is to filter the blood and remove waste products and excess water from the blood. The kidneys process about 200 liters of blood every day and produce about 2 liters of urine. Therefore, when kidney function falls below a certain point, harmful wastes can build up in our body. CKD also triggers other healthcare issues like cardiovascular diseases, which lead to premature death or disability and multiply the amount of money needed for the healthcare of a patient [4]. Unfortunately, there is no cure for CKD, although treatment can slow the progression of the disease. The most common treatment for kidney failure is dialysis. It is estimated that over 2 million people worldwide currently receive treatment with either hemodialysis (HD) or peritoneal dialysis (PD) [3].
Recently, these treatments have been performed at home and easy-to-use machines are being developed for home dialysis. However, these are still an inconvenient, time consuming, and expensive process [5]. Especially in developing countries, economic and manpower factors have limited the regular maintenance HD treatments due to the absence of adequate technical support and frequent power outages, resulting in the death of over 1 million people annually from untreated kidney failure $[3,6]$. Development of more accessible methods for the treatment of kidney failure has been also desired in developed countries because current dialysis system is prone to be damaged by natural disasters such as gigantic earthquakes or tsunami. Indeed, the shortage of dialysate, water, and electricity created a dangerous situation for HDdependent patients after the major earthquake struck northeastern Japan on March 11, 2011 [7]. Therefore, there has been a great need to develop simpler and more accessible methods for the treatment of kidney failure worldwide. 
From these perspectives, we have been developing a nanofiber mesh for the removal of uremic toxins from the blood, which can be incorporated into wearable blood purification systems for kidney failure patients [8]. The nanofiber mesh was fabricated using a versatile and costeffective process called electrospinning. Electrospinning uses electrical forces to produce polymer fibers with diameters ranging from tens of nanometers to several micrometers [9-13]. In early works, electrospinning was limited to the fabrication of nanofibers from organic polymers due to the stringent requirement on the viscoelastic behavior of the electrospinning solution. With the development of electrospinning method and setup, however, electrospun fibers loaded with various nanoparticles and functional molecules can also be prepared if appropriate processing parameters or new designs of setups are employed. We have previously prepared a zeolite-polymer hybrid nanofiber mesh using a blood-compatible polymer, poly(ethylene-co-vinyl alcohol) $(\mathrm{EVOH})$ [8]. The water-insoluble EVOH has hydrophilic vinyl alcohol units [14] which makes it biocompatible material $[15,16]$. In addition, the hydroxyl groups have been used as a reactive site for the further functionalization [17]. Indeed, several papers in the past decade have demonstrated EVOH as a biomaterial. Recent work by Zhu and Sun showed immobilization of chelating groups on the surface of $\mathrm{EVOH}$ nanofiber for selective protein separation [18]. Lou et al., on the other hand, used EVOH membranes to investigate the effects of senescence-associated phenotypic changes on the cellular aging process [15]. EVOH membrane has been also applied to HD patients as a dialyzer membrane and shown minimal platelet activation and little generation of reactive oxygen species by activated neutrophils [19]. Zeolites are aluminosilicates which have microporous structures capable of adsorbing toxins such as creatinine from blood [20-25]. After testing the mesh on its ability to absorb creatinine, we found that a specific ratio of silicon and aluminum within the zeolites was required. Since this approach is based on the principle of "selective adsorption" instead of "diffusion" through a semipermeable membrane, the proposed composite fibers have the potential to be utilized as a new approach to removing nitrogenous waste products from the bloodstream without the requirement of specialized equipment.

The research reported here extends our previous work to improve the creatinine adsorption capacity of the composite nanofiber mesh for practical use. A series of polymer-zeolite compositions was prepared by blending EVOH with zeolite particles. The relationships between zeolite compositions, crystallinity of EVOH, morphology of nanofiber, and their creatinine adsorption ability were, respectively, evaluated. Systematic variations of these parameters will identify key compositional features essential for efficient adsorption of creatinine. We have also tested cytotoxicity of the zeolitepolymer nanofiber mesh using macrophages.

\section{Experimental Section}

2.1. Materials. EVOH with 44 ethylene mol\% (EVAL E105A) was kindly supplied by KURARAY (Okayama, Japan). EVOH with 25 ethylene mol\% (SoarnoL V2504RB) and with 33 ethylene mol\% (GC3304B) were kindly supplied by The Nippon Synthetic Chemical Industry (Osaka, Japan). HSZseries zeolites 320-HOA (USY type), 500-KOA (L), 640-HOA (Mordenite), 720-KOA (Ferrierite), 840-HOA (ZSM-5), and 940-HOA (Beta) were purchased from TOSOH (Yamaguchi, Japan). 1,1,1,3,3,3-Hexafluoroisopropanol (HFIP) was purchased from Sigma Aldrich (Tokyo, Japan). Poly(vinylalcohol) (PVA) and creatinine were purchased from Wako Pure Chemicals (Tokyo, Japan). Alamar blue was purchased from ABD Bioquest (CA, America). Raw 264.7 cells were purchased from InvivoGen (CA, America). Dulbecco's Modified Eagle's Medium (DMEM) was purchased from SIGMA (STL, America). Fetal bovine serum (FBS) was purchased from American Type Culture Collection (VA, America). Antibiotic antimycotic was purchased from Gibco (NY, America). Normocin was purchased from InvivoGen (CA, America).

2.2. Fiber Fabrication. As described previously, EVOH nanofibers were prepared using electrospinning equipment (Nanon-01A; MECC, Fukuoka, Japan) [8]. Briefly, the electrospinning solutions were prepared by dissolving $7 \mathrm{w} / \mathrm{v} \%$ of EVOH in HFIP. Zeolites were then added to the HFIP solution and ultrasonicated in order to disperse the zeolite particles. In this study, 6 different types of zeolite were used as follows: 320-HOA, 500-KOA, 640-HOA, 720-KOA, 840$\mathrm{HOA}$, and 940-HOA. Zeolite to EVOH ratios were varied from $9: 91$ to $47: 53$. The electrospun voltage was $25 \mathrm{kV}$, feed rate was $1.0 \mathrm{~mL} / \mathrm{h}$, and tip to collector distance was $15 \mathrm{~cm}$. A 25-gauge pointed needle was used in all experiments.

2.3. Fiber Characterizations. Scanning electron microscopy (SEM) of the electrospun samples was performed on a Hitachi S-4800 field emission SEM (Hitachi, Tokyo, Japan). Nanofiber samples were sputter-coated with platinum using an E-1030 Ion Sputterer (Hitachi, Tokyo, Japan). The diameter of nanofibers was measured by Image software 40 times. Thermal gravimetric analysis (TG-DTA) of the samples was conducted using TG-DTA6200 under air flow (Seiko Instruments Inc., Chiba, Japan). The temperature scans were taken from room temperature to $500^{\circ} \mathrm{C}$ at $10^{\circ} \mathrm{C} / \mathrm{min}$. The $\mathrm{EVOH}-$ zeolite nanofibers were also examined by energy dispersive $\mathrm{X}$ ray spectroscopy (EDX) equipped in SEM (HORIBA, Kyoto, Japan).

2.4. Adsorption Studies of Creatinine. Adsorption experiments were performed using a batch equilibration technique according to the previously reported method [8]. Briefly, creatinine solution of $190 \mu \mathrm{M}$ concentration was prepared in millipore water. The solution was incubated in the presence of nanofiber mesh for $0,2,5,12$, or $24 \mathrm{~h}$. UV-Visible spectrophotometer (V-650, JASCO, Tokyo, Japan) was used to measure the absorbance of the creatinine solution ( $234 \mathrm{~nm}$ absorption peak). The adsorption experiments were also done at different temperatures $\left(10,25\right.$, or $\left.37^{\circ} \mathrm{C}\right)$ by using a TAITEC PERSONAL-11 water bath shaker (TAITEC, Saitama, Japan). Creatinine adsorption capacity of free zeolites was also tested by the same protocol. 
2.5. Cytotoxic Assay. The cytotoxic assay on the zeolite containing fibers was conducted by Alamar blue assay [26]. Raw 264.7 cells (murine macrophage cells) were seeded on 24well cell culture plates at $1 \times 10^{6}$ cells/well in $1 \mathrm{~mL}$ of DMEM (high glucose D5671 and high glucose D5796) containing 10\% FBS and $0.2 \%$ Normocin. After adding stimulus $(100 \mathrm{mg}$ of nanofiber mesh with $0 \mathrm{wt} \%$ or $9 \mathrm{wt} \%$ zeolite particles and $10 \mathrm{mg}$ of bare zeolite particles), these culture systems were incubated at $37^{\circ} \mathrm{C}$ for $24 \mathrm{~h}$. For measuring cell viability, $100 \mu \mathrm{L}$ of alamer blue dye was added to each well. After incubating at $37^{\circ} \mathrm{C}$ for $4 \mathrm{~h}$, fluorescence was measured (excitation wavelength: $544 \mathrm{~nm}$; fluorescence wavelength: $590 \mathrm{~nm}$ ) using 1420 Multilabel Counter (Perkin Elmer, Massachusetts, America). The relative cytotoxicity for each sample was evaluated by comparing the fluorescent intensities.

\section{Results and Discussion}

3.1. Fabrication of Zeolite/EVOH Nanofiber Mesh. We have previously reported fabrication of zeolite/EVOH nanofiber mesh using an isopropanol or HFIP solvent system [19]. We found that isopropanol solution could only be electrospun for a couple of hours as the EVOH would start to come out of solution. In comparison, the solutions dissolved in HFIP could be electrospun very easily over many days and produced more consistent fibers. Therefore, HFIP solvent system was used for all subsequent experiments in this study. The morphology of the fibers with 940-HOA zeolites was shown in Figure 1(a). Because the average particle size of zeolites was approximately $2 \mu \mathrm{m}$ which is significantly larger than the fiber diameter, bead-like formations were observed. However, continuous fibrous structures were still maintained between the beads. SEM/EDX mapping images of the fibers show the successful incorporation of zeolites within the fibers (Figure 1(b)). Atomic Si was chosen for its presence in zeolites, but not in $\mathrm{EVOH}$. The elemental mapping images of $\mathrm{Si}$ atom (white dots) show that zeolites are successfully incorporated within the fibers. Of particular interest is that uniform distributions of zeolite within the fiber were achieved. This result indicates that zeolite particles with smaller diameters than fiber diameter were incorporated within each fiber. To determine the correlation between incorporated amounts and feeding amounts of zeolites, the elemental mapping images of $\mathrm{Si}$ atom in Figure 1(b) were analyzed using ImageJ software. Figure 2(a) plots the white dots area (zeolite content) against feed concentration. There is a good correlation between incorporated amounts and feeding amounts. TG-DTA testing was further carried out to precisely determine the percentage of zeolites inside the nanofiber meshes (Figure S1 in Supplementary Material available online at http://dx.doi.org/10.1155/2016/5638905). The nanofibers fed with 9,33 , and $47 \mathrm{wt} \%$ zeolite had around 8,35 , and $44 \mathrm{wt} \%$ inside the nanofibers (Figure 2(b)). It was determined that over $90 \%$ of the fed zeolites were successfully incorporated into nanofibers although the deviation between the zeolite in feed and zeolites in fiber became larger with increased zeolite feeding percentages. In our previous report, only $30 \mathrm{wt} \%$ of zeolite was incorporated in the fiber. But we successfully incorporate more than $40 \mathrm{wt} \%$ of zeolite in this study. These results indicated that an EVOH nanofiber composite with higher zeolites contents was successfully fabricated using electrospinning techniques.

3.2. Creatinine Adsorption Capacity of Zeolite/EVOH Nanofiber Mesh. The creatinine adsorption capacities of bare zeolites are shown in Figure S2. The 500-KOA zeolite was nonadsorbent to creatinine. 320-HOA and 720-KOA zeolites were found to have very low creatinine adsorption capacities while the $640-\mathrm{HOA}$ has mid-range $44 \mu \mathrm{mol} / \mathrm{g}$ adsorptions. The zeolite with the greatest creatinine adsorption capacity ( $86 \mu \mathrm{mol} / \mathrm{g}$ ) was found to be the $940-\mathrm{HOA}$ zeolite (Beta type). Therefore, 940-HOA zeolite was chosen for all subsequent experiments in this study. To determine the creatinine adsorption capacities of different composite nanofibers, nanofiber meshes were immersed in creatinine solution of $190 \mu \mathrm{M}$ concentration and incubated at $37^{\circ} \mathrm{C}$ for $12 \mathrm{~h}$. Finally, UV absorption spectrums of solutions collected from the outlet were measured. Figure 3 presents the creatinine adsorption capacity of the composite fibers with various 940 HOA zeolite contents. The creatinine adsorption capacity of the fiber mass was increased by increasing the zeolite content in the fibers (bars in Figure 3). The adsorption capacity of $47 \mathrm{wt} \% 940-\mathrm{HOA}$ sample is as high as $30 \mu \mathrm{mol} / \mathrm{g}$ in $190 \mu \mathrm{M}$ solution. The adsorption capacity of the zeolite mass, on the other hand, was decreased by increasing the zeolite content (circles in Figure 3). In other words, incorporation of large amounts of zeolite into the nanofibers decreases the adsorption efficiency because aggregation of zeolites lowers the effective surface area of zeolites for creatinine adsorption [25]. These results indicate that not only the type of zeolite but also electrospinning conditions have to be carefully chosen to achieve a higher capacity of creatinine adsorption.

We also examined the role of crystallinity of EVOH nanofiber in creatinine adsorption because crystallinity may limit the accessibility of creatinine to zeolites. Adsorption experiments were performed using EVOH nanofibers with different ethylene content. As shown in Figure 4, the ethylene content in EVOH influences adsorption of creatinine. The amount of adsorbed creatinine appeared to increase inversely with ethylene content because nanofiber becomes less accessible at higher degrees of crystallinity. Since temperature is also one of the factors which influence the adsorption of molecules by solids, we examined the temperature dependence of the adsorption capacity (Figure S3). In general, adsorption is thermodynamically more favourable at low temperatures because the bonds between the adsorbate and adsorbent are weakened at higher temperatures. However, the results show that creatinine adsorption capacities of bare zeolites were not affected by temperature, while those of zeolites within the EVOH were significantly decreased with decreasing temperature. These results strongly suggest that temperature affects the crystallinity of EVOH (permeability of creatinine) more than adsorption capacity of zeolite in this study. To further reveal how adsorption time affected the creatinine adsorption capacity, the nanofiber meshes were incubated in creatinine solution of $190 \mu \mathrm{M}$ concentration at $37^{\circ} \mathrm{C}$ for $24 \mathrm{~h}$. Figure 5 shows the mesh adsorbed $50 \mu \mathrm{mol} / \mathrm{g}$ of creatinine in 60 minutes. The creatinine adsorption capacity 

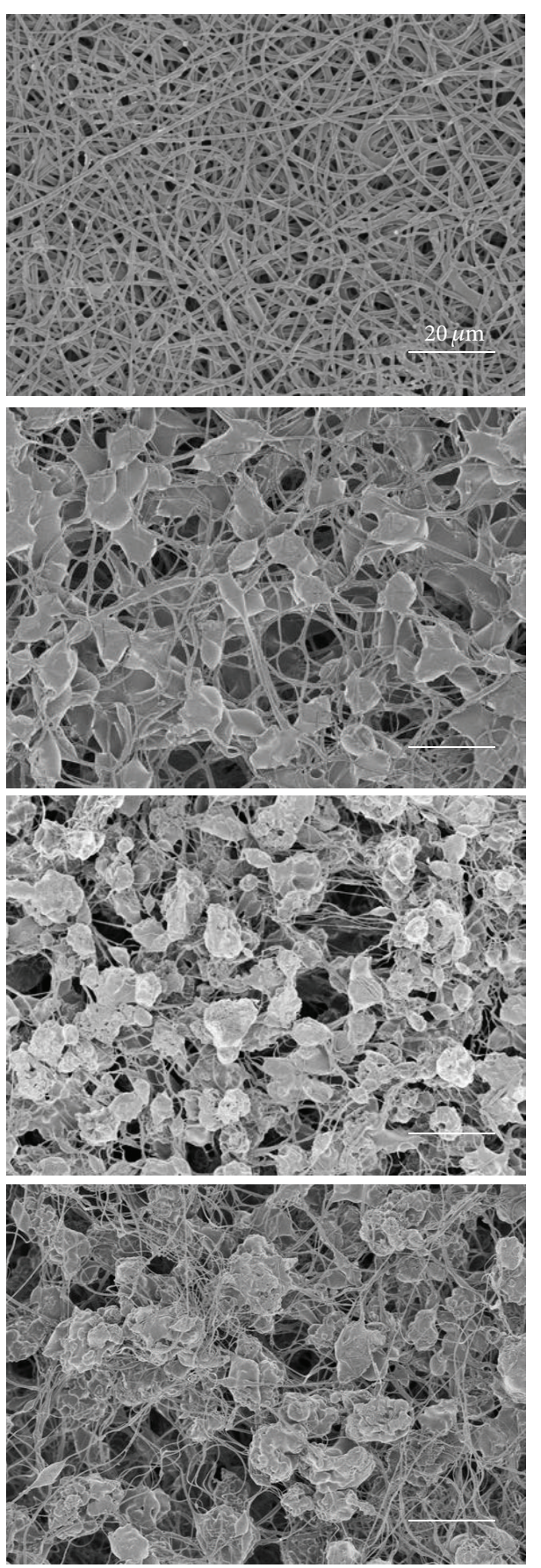

(a)
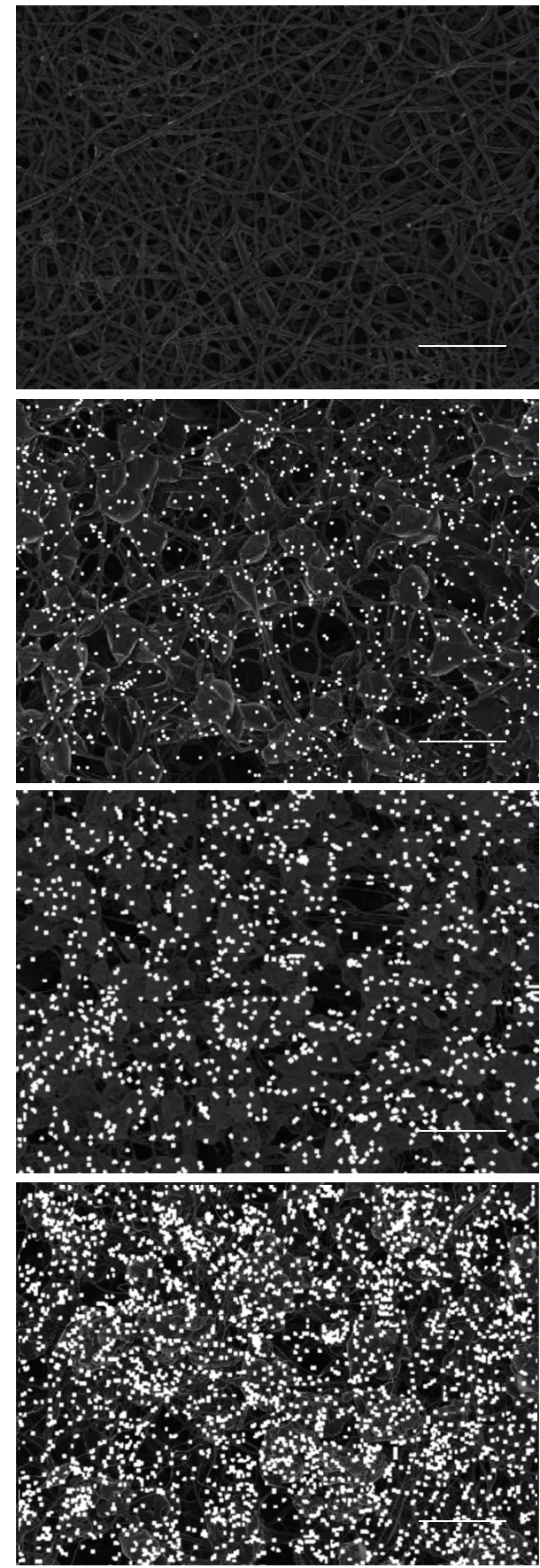

(b)

Figure 1: SEM/EDX images of the EVOH fibers blended with 940-HOA zeolites at different contents ( $0,9,33$, and $47 \mathrm{wt} \%$ in feed) (scale bar: $20 \mu \mathrm{m}$ ). (a) SEM, (b) Si-mapping.

gradually increased with time and the adsorption equilibrium was reached after $12 \mathrm{~h}$.

3.3. Cytotoxicity of Zeolite/EVOH Nanofiber Mesh. Finally, the cytotoxicity of zeolite composite EVOH nanofibers was examined because the possible adverse effects of silicalite particles on human health have long been of concern. In general, the cytotoxicity of silicalite materials is complex and depends on the particle size, surface functional group, and cell line used for the toxicity studies [27]. In this study, we choose RAW264.7 macrophage cell lines because alveolar macrophages play a key role in silica related diseases by clearing particles out of the lung or by causing chronic inflammation. In addition, the RAW264.7 cell line has been 


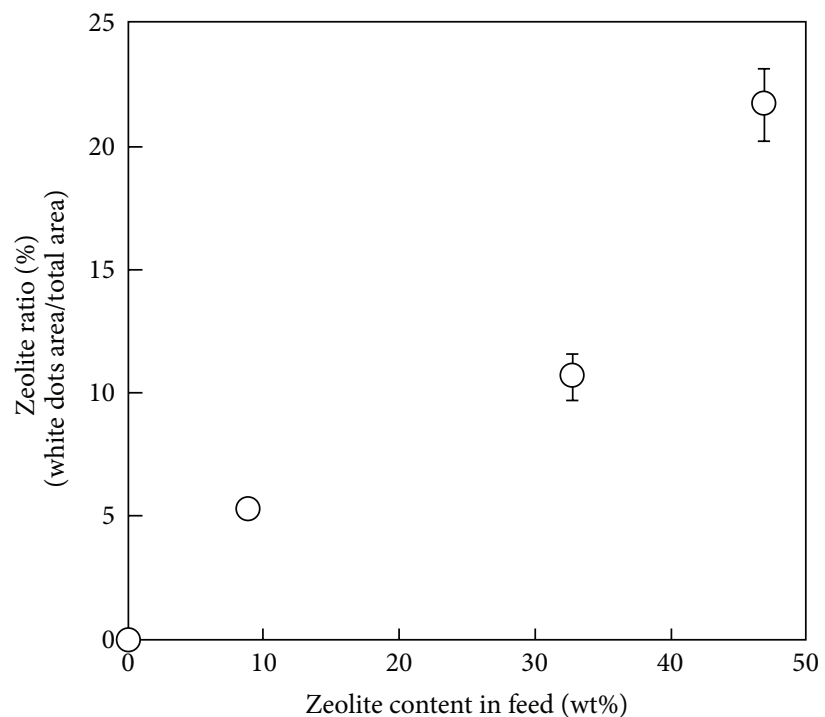

(a)

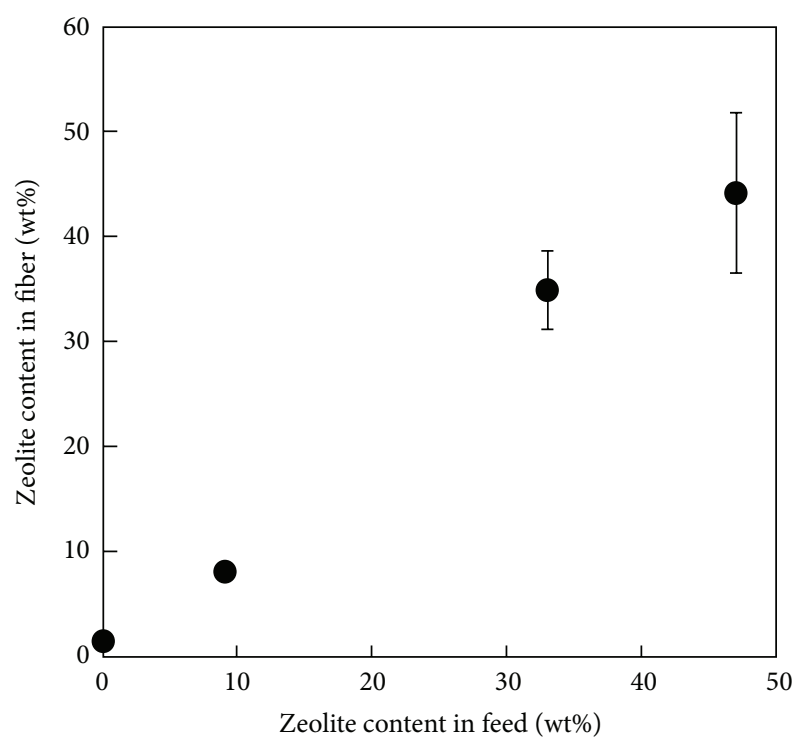

(b)

FIGURE 2: Relationship between zeolite contents in the fiber and their feed concentrations. (a) The zeolite ratios were determined from the white dots area in Si-mapping images $(n=4)$. (b) The zeolite contents were calculated from the weight percentages of zeolites in nanofibers by TG-DTA testing under air flow. The weight percentages of zeolites in nanofibers were calculated from the weight loss of nanofiber composites $(n=3)$.

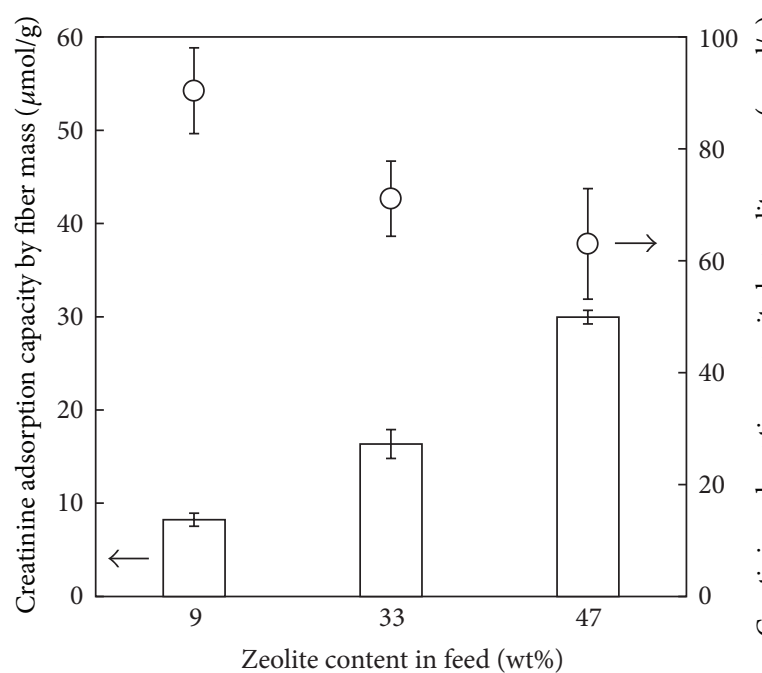

Figure 3: Creatinine adsorption capacity of EVOH nanofibers incorporated with 940 -HOA zeolite by fiber mass (bars) and zeolite mass (circles). Immersion time was $12 \mathrm{~h}$ at $37^{\circ} \mathrm{C}(n=3)$.

known as a model macrophage cell line commonly used to represent the physiological scavengers of foreign nanoparticles. A comparison of the cytotoxicity of bare 940-HOA zeolite particles and incorporated 940-HOA zeolite particles within EVOH nanofiber is shown in Figure 6. The nanofiber composite was relatively nontoxic with a cell viability of approximately $90 \%$. These phenomena can be explained by the fact that zeolite particles were successfully encapsulated in the EVOH fiber, preventing perturbation of the cell membrane as well as cellular uptake. On the other hand,

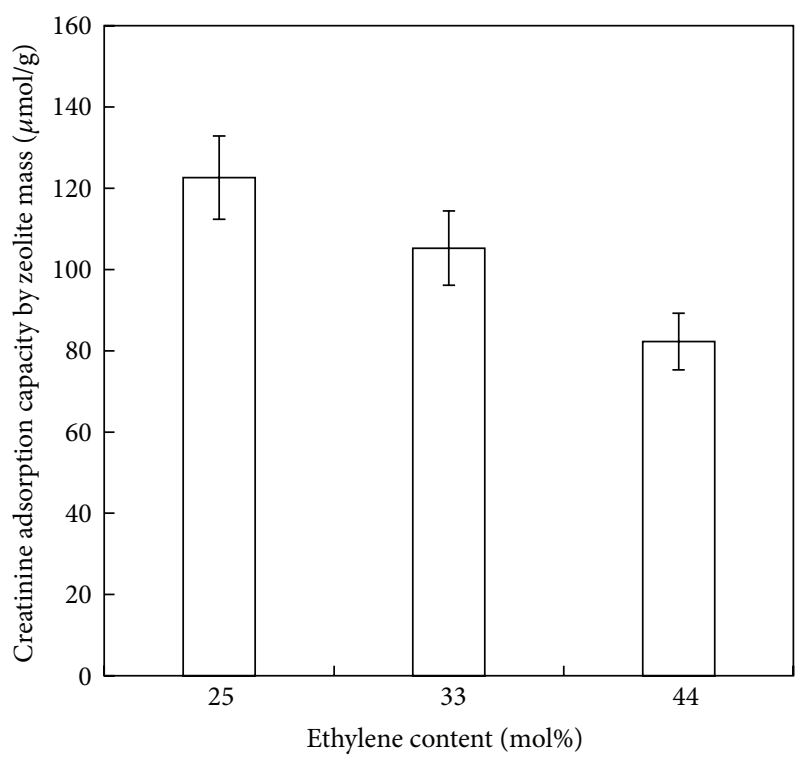

FIGURE 4: Creatinine adsorption capacity of the EVOH nanofibers incorporated with $940-\mathrm{HOA}$ zeolite as a role of ethylene content in EVOH (after $12 \mathrm{~h}$ of immersion at $\left.37^{\circ} \mathrm{C}\right)(n=3)$.

bare zeolites were surprisingly toxic at this concentration level, although zeolites are thought to be safe materials. This is mainly because concentration ranges which have been demonstrated in the previous study are $50-100 \mu \mathrm{g} / \mathrm{mL}$ range which is significantly lower than that of our study $(10 \mathrm{mg} / \mathrm{mL})$. Thus, the current experimental results suggest that zeolite/EVOH nanofiber meshes can be useful as the 


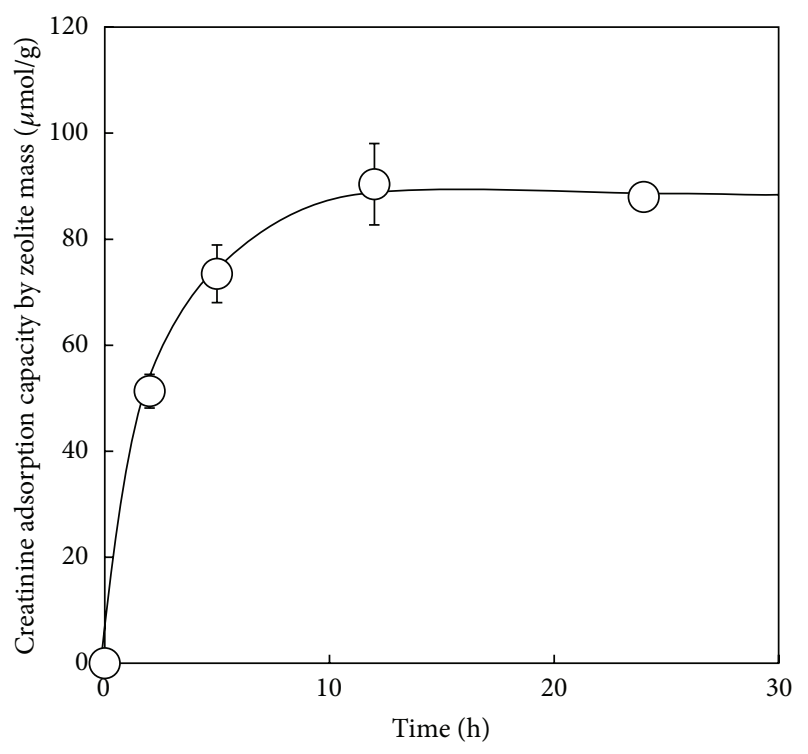

FIGURE 5: Creatinine adsorption capacity of the zeolite/EVOH nanofiber meshes as a role of immersion time at $37^{\circ} \mathrm{C}(n=3)$.

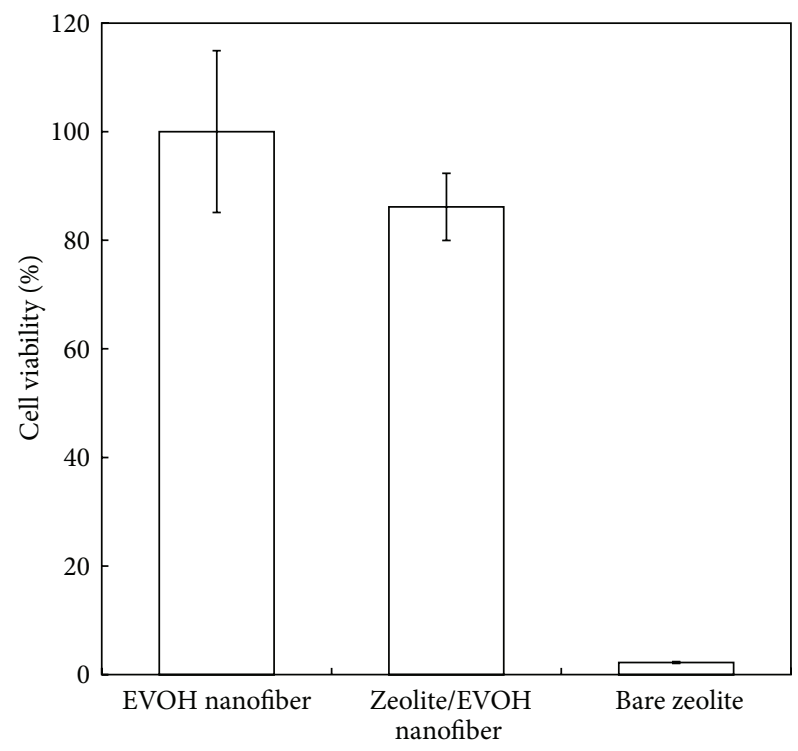

FIGURE 6: The relativity of cytotoxicity in Raw 264.7 cells. Cells were incubated at $37^{\circ} \mathrm{C}$ for $24 \mathrm{~h}$ with $\mathrm{EVOH}$ nanofiber, zeolite/EVOH nanofiber, and bare zeolite particles.

safe and effective alternative of blood purification therapy in future clinical approach.

\section{Conclusions}

This study demonstrated the rational strategy of fabrication of zeolite/EVOH nanofiber meshes for the efficient removal of creatinine. Combined with SEM/EDX and TG-DTA analysis, we found that over $90 \%$ of fed zeolites were successfully incorporated into nanofibers. The amount of adsorbed creatinine onto the incorporated zeolites appeared to increase inversely with ethylene content of EVOH because nanofiber becomes less accessible at higher degrees of crystallinity. Cytotoxic assay revealed that the zeolite/EVOH nanofiber was relatively nontoxic because EVOH layers prevented zeolite particles from direct contact with cells. These results suggest that the nanofibrous structure provides extremely large surface area and porosity while encapsulation of zeolite particles prevents the direct contact with cells. Thus, the rational design of hybridization of absorbent (zeolite) and biocompatible matrix $(\mathrm{EVOH})$ will pave the way for efficient removal of uremic toxins from the blood as a wearable blood purification system. The explored scientific data needs further investigation for long term exposure results to blood sample, which will be useful for future clinical applications.

\section{Competing Interests}

The authors declare that they have no competing interests.

\section{Acknowledgments}

This work was partially supported by JSPS KAKENHI (16K01402 and 26750152).

\section{References}

[1] V. Jha, G. Garcia-Garcia, K. Iseki et al., "Chronic kidney disease: global dimension and perspectives," The Lancet, vol. 382, no. 9888, pp. 260-272, 2013.

[2] L. J. Hickson, A. Eirin, and L. O. Lerman, "Challenges and opportunities for stem cell therapy in patients with chronic kidney disease," Kidney International, vol. 89, no. 4, pp. 767-778, 2016.

[3] W. G. Couser, G. Remuzzi, S. Mendis, and M. Tonelli, "The contribution of chronic kidney disease to the global burden of major noncommunicable diseases," Kidney International, vol. 80, no. 12, pp. 1258-1270, 2011.

[4] A. C. Schoolwerth, M. M. Engelgau, T. H. Hostetter et al., "Chronic kidney disease: a public health problem that needs a public health action plan," Preventing Chronic Disease, vol. 3, no. 2, p. A57, 2006.

[5] B. A. Young, C. Chan, C. Blagg et al., "How to overcome barriers and establish a successful home HD program," Clinical Journal of the American Society of Nephrology, vol. 7, no. 12, pp. 20232032, 2012.

[6] M. El Nahas, “The global challenge of chronic kidney disease," Kidney International, vol. 68, no. 6, pp. 2918-2929, 2005.

[7] M. Nangaku and T. Akizawa, "Diary of a Japanese nephrologist during the present disaster," Kidney International, vol. 79, no. 10, pp. 1037-1039, 2011.

[8] K. Namekawa, M. T. Schreiber, T. Aoyagi, and M. Ebara, "Fabrication of zeolite-polymer composite nanofibers for removal of uremic toxins from kidney failure patients," Biomaterials Science, vol. 2, no. 5, pp. 674-679, 2014.

[9] X. Shi, W. Zhou, D. Ma et al., "Electrospinning of nanofibers and their applications for energy devices," Journal of Nanomaterials, vol. 2015, Article ID 140716, 20 pages, 2015.

[10] Y.-J. Kim, M. Ebara, and T. Aoyagi, “Temperature-responsive electrospun nanofibers for 'on-off' switchable release of dextran," Science and Technology of Advanced Materials, vol. 13, no. 6, Article ID 064203, 2012. 
[11] R. Garrett, E. Niiyama, Y. Kotsuchibashi, K. Uto, and M. Ebara, "Biodegradable nanofiber for delivery of immunomodulating agent in the treatment of basal cell carcinoma," Fibers, vol. 3, no. 4, pp. 478-490, 2015.

[12] H.-L. Che, H. J. Lee, K. Uto et al., "Simultaneous drug and gene delivery from the biodegradable poly( $\varepsilon$-caprolactone) nanofibers for the treatment of liver cancer," Journal of Nanoscience and Nanotechnology, vol. 15, no. 10, pp. 7971-7975, 2015.

[13] T. Okada, E. Niiyama, K. Uto, T. Aoyagi, and M. Ebara, "Inactivated Sendai virus (HVJ-E) immobilized electrospun nanofiber for cancer therapy," Materials, vol. 9, no. 1, article 12, 2016.

[14] K. K. Mokwena and J. Tang, "Ethylene vinyl alcohol: a review of barrier properties for packaging shelf stable foods," Critical Reviews in Food Science and Nutrition, vol. 52, no. 7, pp. 640650, 2012.

[15] P.-J. Lou, M.-Y. Chiu, C.-C. Chou, B.-W. Liao, and T.-H. Young, "The effect of poly (ethylene-co-vinyl alcohol) on senescenceassociated alterations of human dermal fibroblasts," Biomaterials, vol. 31, no. 7, pp. 1568-1577, 2010.

[16] T.-H. Young, C.-W. Lin, L.-P. Cheng, and C.-C. Hsieh, "Preparation of EVAL membranes with smooth and particulate morphologies for neuronal culture," Biomaterials, vol. 22, no. 13, pp. 1771-1777, 2001.

[17] Y. Kotsuchibashi and M. Ebara, "Facile functionalization of electrospun poly(ethylene-co-vinyl alcohol) nanofibers via the benzoxaborole-diol interaction," Polymers, vol. 8, article 2, 2016.

[18] J. Zhu and G. Sun, "Facile fabrication of hydrophilic nanofibrous membranes with an immobilized metal-chelate affinity complex for selective protein separation," ACS Applied Materials \& Interfaces, vol. 6, no. 2, pp. 925-932, 2014.

[19] Y. Matsumoto, M. Mukai, K. Arihara, T. Saito, and H. Kumagai, "Ethylene-vinyl alcohol copolymer dialyzer membrane reduces protein oxidation in hemodialysis patients," Renal Failure, vol. 33, no. 4, pp. 382-387, 2011.

[20] D. Bergé-Lefranc, H. Pizzala, J. L. Paillaud et al., "Adsorption of small uremic toxin molecules on MFI type zeolites from aqueous solution," Adsorption, vol. 14, no. 2-3, pp. 377-387, 2008.

[21] D. Bergé-Lefranc, H. Pizzala, R. Denoyel et al., "Mechanism of creatinine adsorption from physiological solutions onto mordenite," Microporous and Mesoporous Materials, vol. 119, no. 1-3, pp. 186-192, 2009.

[22] D. Bergé-Lefranc, O. Schäf, R. Denoyel et al., "The extraction of creatinine from a physiological medium by a microporous solid and its quantification by diffuse reflectance UV spectroscopy," Microporous and Mesoporous Materials, vol. 129, no. 1-2, pp. 144-148, 2010.

[23] D. Bergé-Lefranc, C. Vagner, R. Calaf et al., "In vitro elimination of protein bound uremic toxin p-cresol by MFI-type zeolites," Microporous and Mesoporous Materials, vol. 153, pp. 288-293, 2012.

[24] V. Wernert, O. Schäf, H. Ghobarkar, and R. Denoyel, "Adsorption properties of zeolites for artificial kidney applications," Microporous and Mesoporous Materials, vol. 83, no. 1-3, pp. 101113, 2005.

[25] L. Lu, C. Samarasekera, and J. T. W. Yeow, "Creatinine adsorption capacity of electrospun polyacrylonitrile (PAN)-zeolite nanofiber membranes for potential artificial kidney applications," Journal of Applied Polymer Science, vol. 132, no. 34, 2015.

[26] M. G. Gutierrez, S. S. Master, S. B. Singh, G. A. Taylor, M. I. Colombo, and V. Deretic, "Autophagy is a defense mechanism inhibiting BCG and Mycobacterium tuberculosis survival in infected macrophages," Cell, vol. 119, no. 6, pp. 753-766, 2004.

[27] T. Kihara, Y. Zhang, Y. Hu, Q. Mao, Y. Tang, and J. Miyake, "Effect of composition, morphology and size of nanozeolite on its in vitro cytotoxicity," Journal of Bioscience and Bioengineering, vol. 111, no. 6, pp. 725-730, 2011. 

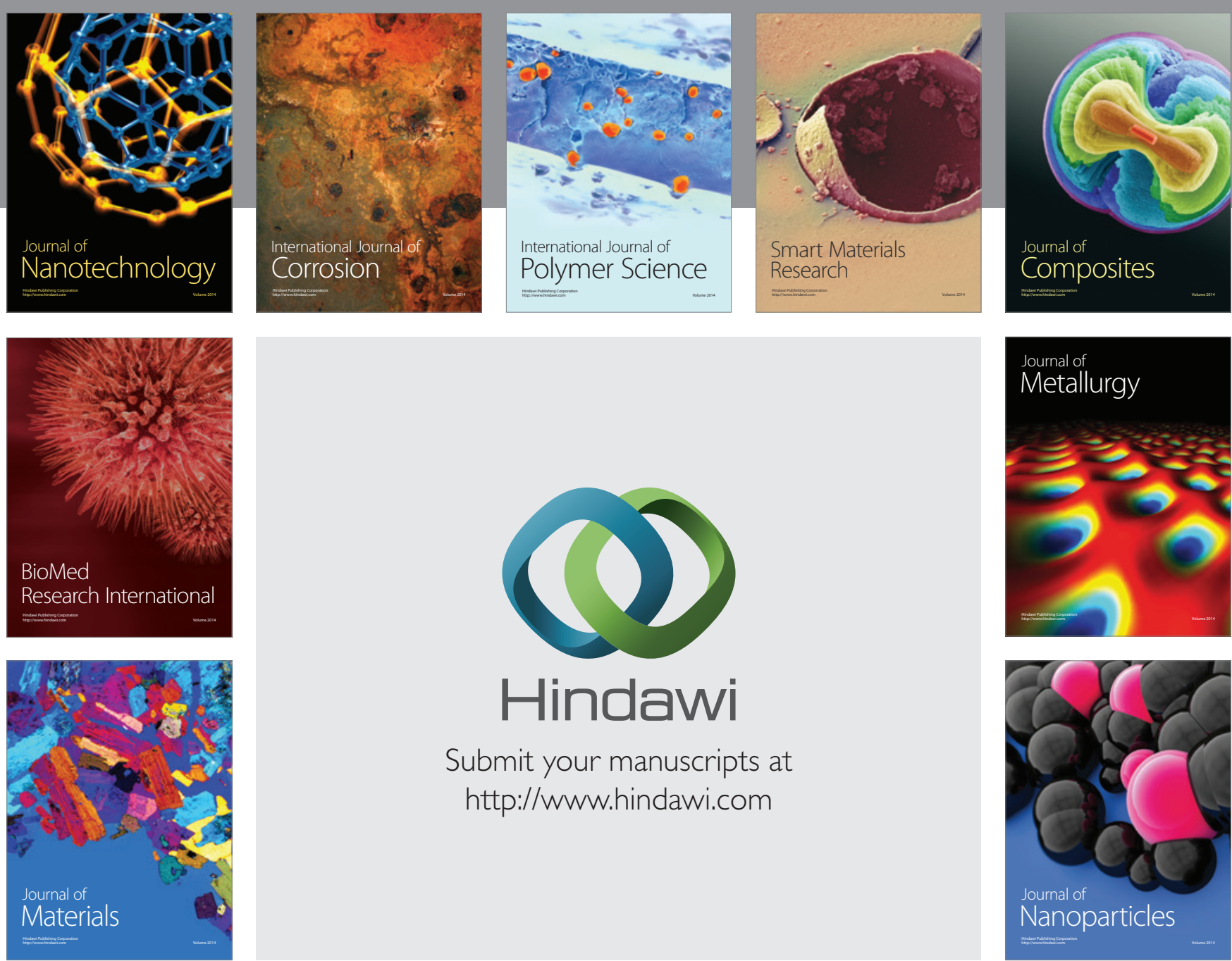

\section{Hindawi}

Submit your manuscripts at

http://www.hindawi.com

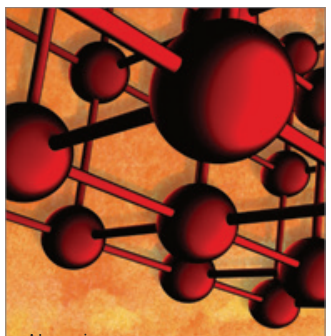

Materials Science and Engineering
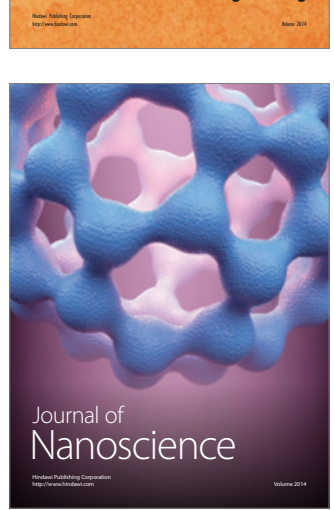
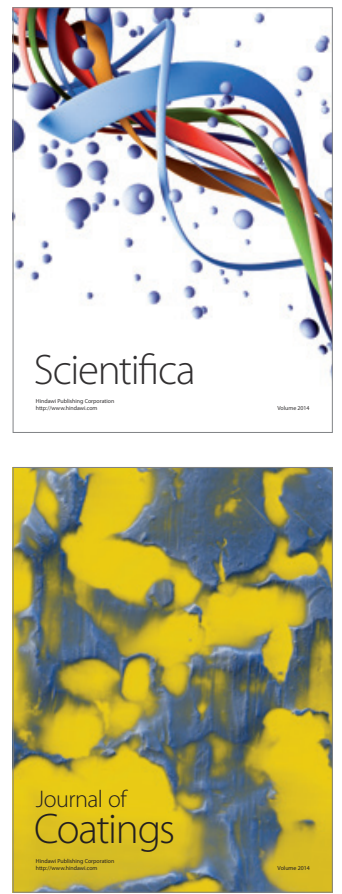
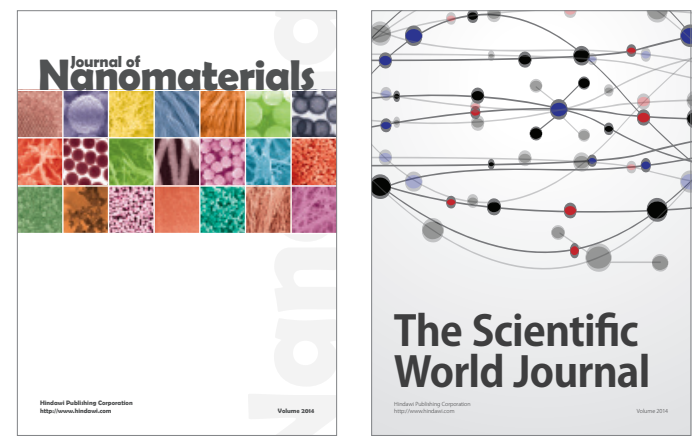

The Scientific World Journal
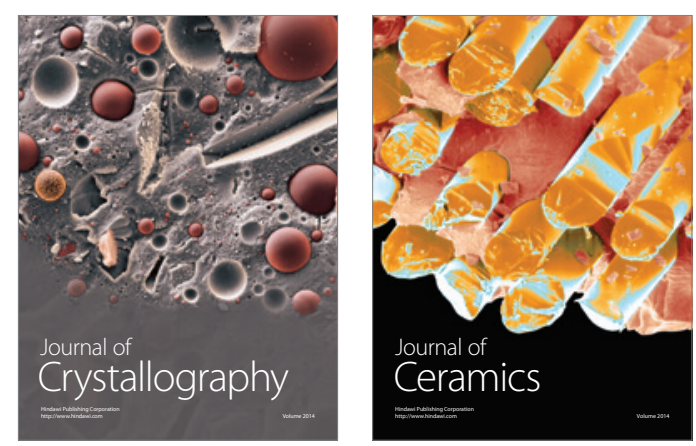
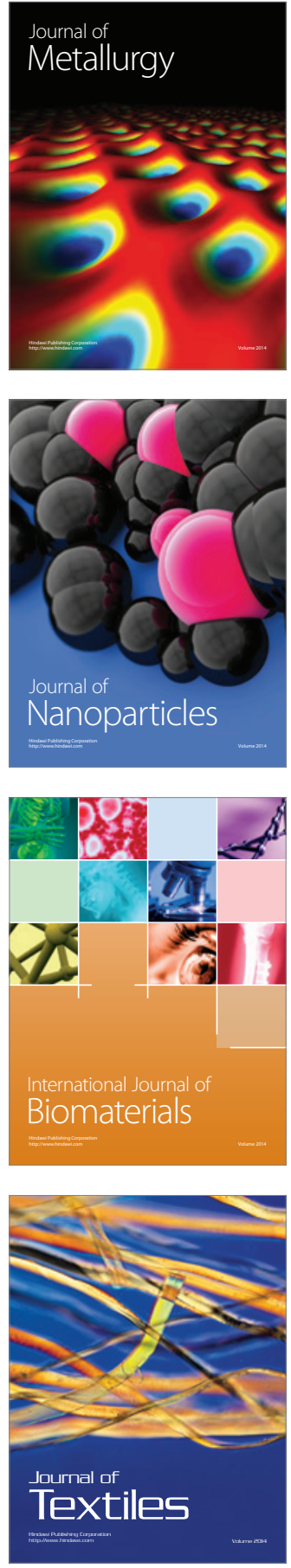